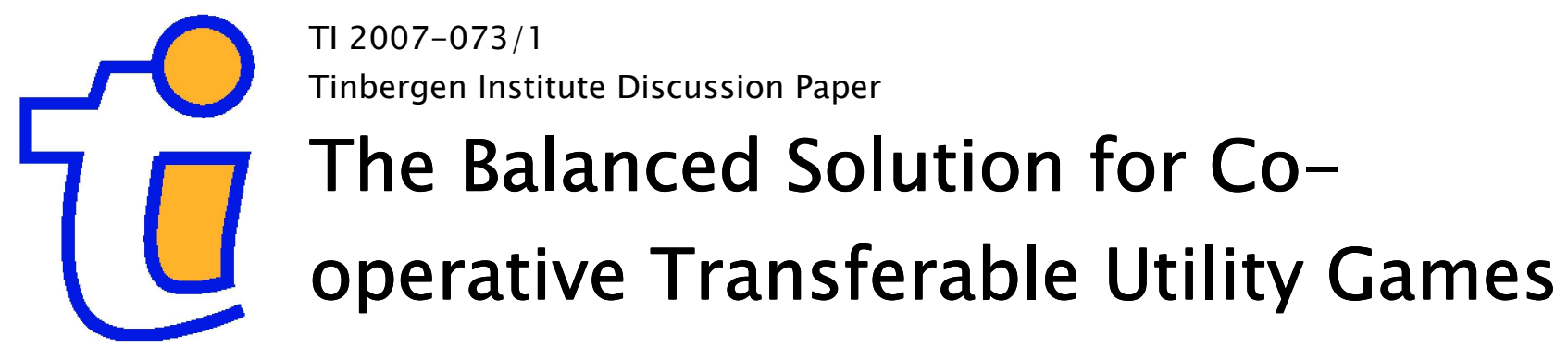

René van den Brink ${ }^{1}$

René Levínsky²

Miroslav Zeleny3

I Free University Amsterdam, and Tinbergen Institute, the Netherlands;

2 Max Planck Institute of Economics, Jena, Germany;

${ }^{3}$ Charles University, Prague, Czech Republic. 


\section{Tinbergen Institute}

The Tinbergen Institute is the institute for economic research of the Erasmus Universiteit Rotterdam, Universiteit van Amsterdam, and Vrije Universiteit Amsterdam.

Tinbergen Institute Amsterdam

Roetersstraat 31

1018 WB Amsterdam

The Netherlands

Tel.: $\quad+31(0) 205513500$

Fax: $\quad+31(0) 205513555$

Tinbergen Institute Rotterdam

Burg. Oudlaan 50

3062 PA Rotterdam

The Netherlands

Tel.: $\quad+31(0) 104088900$

Fax: $\quad+31(0) 104089031$

Most TI discussion papers can be downloaded at http:/ /www.tinbergen.nl. 


\title{
The balanced solution for cooperative transferable utility games
}

\author{
René van den Brink \\ Department of Econometrics and Tinbergen Institute, \\ Faculty of Economics and Business Administration, Free University, \\ De Boelelaan 1105, 1081 HV Amsterdam, The Netherlands, \\ René Levínský* \\ Max Planck Institute of Economics, \\ Kahlaische Straße 10, 07745 Jena, Germany, \\ and Miroslav Zelený ${ }^{\dagger}$ \\ Department of Mathematical Analysis, \\ Faculty of Mathematics and Physics, Charles University, \\ Sokolovská 83, 18675 Praha, Czech Republic.
}

October 10, 2007

\begin{abstract}
:
The Shapley value of a cooperative transferable utility game distributes the dividend of each coalition in the game equally among its members. Given exogenous weights for all players, the corresponding weighted Shapley value distributes the dividends proportionally to their weights. In this contribution we define the balanced solution which assigns weights to players such that the corresponding weighted Shapley value of each player is equal to her weight. We prove its existence for all monotone transferable utility games, discuss other properties of this solution, and deal with its characterization through a reduced game consistency.
\end{abstract}

JEL Classification: C71

Key words: Balanced solution, Proportionality, Reduced game consistency, Weighted Shapley value.

${ }^{*}$ Corresponding author: levinsky@econ.mpg.de, Phone: +49-3641-686 642, Fax: +49-3641-686 667.

${ }^{\dagger}$ The author was supported by the research project MSM 0021620839 financed by MSMT 


\section{INTRODUCTION}

Consider a finite set $N$ with $n$ players. Situations where each subset of players of $N$ can generate a certain worth can be described by a cooperative transferable utility game (or simply $T U$-game) $(N, v)$ : for any coalition $S \subseteq N$, the real number $v(S)$ is the worth of $S$, which the members of the coalition $S$ can distribute among themselves. A payoff vector in an $n$-player TU-game is an $n$-dimensional vector whose components are the payoffs of the corresponding players. A single-valued solution for a class $\mathcal{C}$ of TU-games is a function that assigns a payoff vector to every TU-game in $\mathcal{C}$. The best known single-valued solution for TU-games is the Shapley value, Shapley (1953b), which distributes the so-called Harsanyi dividends of the game equally among the players in the corresponding coalitions (see Section 2 for undefined notions).

The equal distribution of the dividends seems questionable in situations that suggest proportionality rather than equality. A prominent example is the sociopsychological equity theory of Homans (1961) and Selten (1978). The standard business practice of dividing a firm's profit proportionally to investment (constant return per share) could serve as a daily-life example of the same phenomenon. (Subadditive) cost games provide another example. Consider a market with non-linear pricing where the unit price depends on the purchased volume, e.g., it equals to 10 for quantities bellow five and 8 for higher amounts. Suppose there are two agents in the market who have to buy two and three units, respectively. One might expect that the buyers will agree to pool their resources and buy together the five units to guarantee the lower price for both of them, spending 16 and 24 units, respectively. On the contrary, all prominent solutions for TU-games (Shapley value, nucleolus etc.) are based on equal split of the dividends for 2-person games. In this example these solutions predict that buyers split the cost savings (of 10) equally (spending 15 and 25 respectively, and facing different prices of $15 / 2$ and $25 / 3$ ).

Several authors have proposed 'proportional' solutions for particular classes of games (see, e.g., Kalai, 1977; Roth, 1979; Hart and Mas-Colell, 1989; Feldman, 2005; Ortmann, 2000). In this paper we modify the so-called proper Shapley value, 
the solution proposed by Vorob'ev and Liapounov (1998). Their approach is based on the weighted Shapley value, Shapley (1953a), where the dividends are distributed among players proportionally according to some exogenously given weights. The proper Shapley value distributes the worth of the grand coalition $N$ among the players in such a way that the payoff vector $x$ coincides with the weighted Shapley value with respect to the weight scheme given by that vector $x$. Thus the proper Shapley value is obtained as a fixed point of the mapping that appears in the definition of the weighted Shapley value.

Vorob'ev and Liapounov (1998) proved the existence of the proper Shapley value for all games with nonnegative dividends. These games form a proper subclass of monotone, convex games. Similarly to their approach, we consider fixed points of a particular mapping on the payoff simplex. Our mapping coincides with that of Vorob'ev and Liapounov for positive efficient weights; for weights on the boundary of the nonnegative efficient simplex we follow the original Shapley value approach and split the dividends equally among the players in the corresponding coalition. A payoff vector obtained in this way will be called a balanced value. The balanced solution assigns to each game the set of all balanced values. The idea behind the proper Shapley value and our solution is identical: since the payoff vector given by the weighted Shapley value and the vector of weights itself express the power of the players in the game, they should naturally coincide.

The paper is organized as follows. In Section 2 we present some basic facts about TU-games and their solutions. The balanced value and the balanced solution are introduced in Section 3. We discuss some basic properties and state the theorem saying that each monotone game admits at least one balanced value. We also deal with a characterization of the balanced solution adapting the reduced game property used by Hart and Mas-Colell $(1988,1989)$ to axiomatize the Shapley value. Finally, the proofs of the main results are presented in Section 4.

\section{Preliminaries}

Let us start with several formal definitions. A transferable utility game (TU-game for short) is a pair $(N, v)$ where $N=\{1, \ldots, n\}$ and $v$ is a characteristic function 
assigning to each subset $S \subseteq N$ a real number $v(S)$ whereas $v(\emptyset)=0$. We denote the collection of all TU-games by $\mathcal{G}$.

A TU-game $(N, v)$ is

- monotone if $v(S) \leq v(T)$ whenever $S \subseteq T \subseteq N$,

- superadditive if $v(S)+v(T) \leq v(S \cup T)$ whenever $S, T \subseteq N$ are disjoint.

Let $(N, v) \in \mathcal{G}$. The dividends $\Delta_{N, v}(S), S \subseteq N$, are defined inductively by

$$
\Delta_{N, v}(S)= \begin{cases}0, & \text { if } S=\emptyset, \\ v(S)-\sum_{T \subsetneq S} \Delta_{N, v}(T), & \text { if } S \neq \emptyset\end{cases}
$$

(see Harsanyi, 1959). Let us note that $v(S)=\sum_{T \subseteq S} \Delta_{N, v}(T)$ for every $S \subseteq N$. This formula shows that the dividends uniquely determine the characteristic function.

We employ the following notation. Let $N$ be a finite nonempty set, $y \in \mathbb{R}^{N}$, and $S \subseteq N$. The symbol $\left.y\right|_{S}$ stands for the restriction of $y$ to $S$ and $y_{S}$ stands for $\sum_{i \in S} y_{i}$ whereas $y_{\emptyset}=0$.

A payoff vector $x \in \mathbb{R}^{N}$ for a game $(N, v)$ is efficient if it exactly distributes worth $v(N)$ of the grand coalition $N$, i.e., if $x_{N}=v(N)$. The set of all efficient payoff vectors of $(N, v)$ is denoted by $X(N, v)$ and the set of all efficient payoff vectors with positive coordinates is denoted by $X_{+}(N, v)$. If there is no danger of confusion we write simply $X$ and $X_{+}$instead of $X(N, v)$ and $X_{+}(N, v)$.

Let $\mathcal{C} \subseteq \mathcal{G}$. A single-valued solution on $\mathcal{C}$ is a function $f$ that assigns to every game $(N, v) \in \mathcal{C}$ a payoff vector $f(N, v)$. A single-valued solution $f$ is efficient on $\mathcal{C}$ if $f(N, v)$ is an efficient payoff vector for all $(N, v) \in \mathcal{C}$. A set-valued solution $F$ on $\mathcal{C}$ assigns a set of payoff vectors $F(N, v)$ to every game $(N, v) \in \mathcal{C}$. A set-valued solution $F$ is efficient on $\mathcal{C}$ if every payoff vector in $F(N, v)$ is efficient whenever $(N, v) \in \mathcal{C}$.

The Shapley value (Shapley, 1953a) of a game $(N, v)$ is an efficient single-valued solution obtained by distributing the dividends of every coalition equally among all players in the coalition, i.e., it is the function $\varphi: \mathcal{G} \rightarrow \mathbb{R}^{N}$ defined by

$$
\varphi_{i}(N, v)=\sum_{\substack{S \subseteq N \\ i \in S}} \frac{1}{|S|} \Delta_{N, v}(S), \quad i \in N,
$$

where $|S|$ denotes the cardinality of $S$. 
Given a weight vector $\omega \in \mathbb{R}^{N}$ with weights $\omega_{i}>0, i \in N$, the corresponding weighted Shapley value (Shapley, 1953b) is the function $\varphi^{\omega}: \mathcal{G} \rightarrow \mathbb{R}^{N}$ defined by

$$
\varphi_{i}^{\omega}(N, v)=\sum_{\substack{S \subseteq N \\ i \in S}} \frac{\omega_{i}}{\omega_{S}} \Delta_{N, v}(S), \quad i \in N .
$$

The weighted Shapley value thus distributes the dividends of coalitions proportionally to the exogenously given weights of the players. Clearly, if all weights $\omega_{i}$ are equal to each other then the weighted Shapely value $\varphi^{\omega}(N, v)$ is equal to the Shapley value $\varphi(N, v)$. Further observe that if $\omega$ and $\widetilde{\omega}$ are positive weight vectors with $\widetilde{\omega}_{i} / \widetilde{\omega}_{j}=\omega_{i} / \omega_{j}$ for all $i, j \in N$ then $\varphi^{\omega}(N, v)=\varphi^{\tilde{\omega}}(N, v)$.

The best known set-valued solution is the core which assigns to every game the set of efficient payoff vectors that are group stable in the sense that every coalition gets at least its own worth. So, the core of a game $(N, v)$ is the set of payoff vectors given by

$$
\operatorname{Core}(N, v)=\left\{x \in \mathbb{R}^{N} \mid x_{N}=v(N) \text { and } x_{S} \geq v(S) \text { for all } S \subseteq N\right\}
$$

A problem with the core is that it can be empty, even for monotone superadditive games.

Let us recall several notions which will be helpful later.

Definition 1. Let $(N, v)$ be a TU-game.

- A component in $(N, v)$ is a coalition $C \subseteq N$ such that

$$
v(S)=v(S \backslash C)+v(S \cap C)
$$

for all $S \subseteq N$ (see, e.g., Aumann and Drèze, 1980, Chang and Kan, 1994).

- Player $i \in N$ is a null player in $(N, v)$ if $v(S)=v(S \backslash\{i\})$ for all $S \subseteq N$.

- Players $i, j \in N$ are symmetric with respect to $(N, v)$ if, for each $S \subseteq$ $N \backslash\{i, j\}$, we have $v(S \cup\{i\})=v(S \cup\{j\})$.

In the next definition we recall some notions related to solutions of TU-games.

Definition 2. Let $\mathcal{C} \subseteq \mathcal{G}$ be a class of games and $F$ be a solution defined on $\mathcal{C}$. A solution $F$ satisfies on $\mathcal{C}$ 
- component efficiency if, for every $(N, v) \in \mathcal{C}, x \in F(N, v)$, and every component $C$ in $(N, v)$, we have $x_{C}=v(C)$;

- the component restriction property if, for every $(N, v) \in \mathcal{C}$, every component $C$ in $(N, v)$, and every $x, x^{\prime} \in F(N, v)$, we have that $x^{\prime \prime}$ given by

$$
x_{i}^{\prime \prime}= \begin{cases}x_{i} & \text { for } i \in C, \\ x_{i}^{\prime} & \text { for } i \in N \backslash C,\end{cases}
$$

is an element of $F(N, v)$;

- the null player property if, for every $(N, v) \in \mathcal{C}$ and $x \in F(N, v)$, we have $x_{i}=0$ whenever $i$ is a null player in the game $(N, v)$;

- the symmetry property if, for every $(N, v) \in \mathcal{C}$ and $x \in F(N, v)$, we have $x_{i}=x_{j}$ whenever $i$ and $j$ are symmetric players with respect to $(N, v)$;

- individual rationality, if $x_{i} \geq v(\{i\})$ for every $(N, v) \in \mathcal{C}, x \in F(N, v)$, and $i \in N$.

\section{BAlANCED VAlues, BAlANCED solution, AND THEIR PROPERTiES}

Let $(N, v)$ be a given game and $\omega^{1} \in X_{+}$be an initial weight scheme where the weights equal each other. Applying the Shapley value (1) we get a redistributed weight vector $\omega^{2}:=\varphi(N, v)$ reflecting the power of the players. Thus it seems natural to consider $\omega^{2}$ as a new weight scheme for players in the game $(N, v)$. Applying the weighted Shapley value repeatedly we get a sequence $\left(\omega^{k}\right)_{k=1}^{\infty}$ of weights satisfying $\omega^{k+1}=\varphi^{\omega^{k}}(N, v)$ (assuming $\omega^{k}$ to have strictly positive coordinates). Obviously, the 'limit weights' (if such exist) will be invariant to the redistribution mechanism. This turns our attention to the fixed points of the mapping $\omega \mapsto \varphi^{\omega}(N, v)$.

Let us be more formal. Let $(N, v) \in \mathcal{G}$ and define $h(N, v): X \rightarrow \mathbb{R}^{N}$ by

$$
h(N, v)_{i}(x)=\sum_{\substack{S \subseteq N, i \in S \\ x_{S} \neq 0}} \frac{x_{i}}{x_{S}} \Delta_{N, v}(S)+\sum_{\substack{S \subseteq N, i \in S \\ x_{S}=0}} \frac{1}{|S|} \Delta_{N, v}(S), \quad i \in N .
$$

For the sake of brevity, we omit the parameters $(N, v)$ if no confusion is possible.

The mapping $h$ coincides with the mapping $\omega \mapsto \varphi^{\omega}(N, v)$ on $X_{+}$. The second sum in the definition of $h$ is important when dealing with zero weights. Since we cannot divide by $x_{S}$ if $x_{S}=0$, we follow the original Shapley value approach and 
split the dividends equally among the players in the corresponding coalition in that case.

Remark 1 . For every $x \in X$ we have

$$
\begin{aligned}
h_{N}(x) & =\sum_{i \in N} h_{i}(x)=\sum_{\substack{S \subseteq N \\
x_{S} \neq 0}} \Delta_{N, v}(S)+\sum_{\substack{S \subseteq N \\
x_{S}=0}} \Delta_{N, v}(S)=\sum_{S \subseteq N} \Delta_{N, v}(S) \\
& =v(N) .
\end{aligned}
$$

This means that $h$ maps values from $X$ to $X$.

The next definition introduces the key notion of our paper.

Definition 3. Let $(N, v) \in \mathcal{G}$. A vector $x \in X$ is called a balanced value if $h(x)=x$ and $x_{i} \geq 0$ for all $i \in N$.

We denote

$$
B(N, v)=\{x \in X(N, v) \mid x \text { is a balanced value of }(N, v)\} .
$$

Remark 2. (i) We require the balanced values to be nonnegative since we consider them as payoff vectors as well as weight schemes.

(ii) Consider a two-player game $(N, v)$, where $N=\{1,2\}$ and $v$ satisfies $v(\{1\})>$ $0, v(\{2\})>0$, and $v(N)>0$. Then a straightforward computation gives a (unique) balanced value

$$
\left(\frac{v(\{1\})}{v(\{1\})+v(\{2\})} \cdot v(N), \frac{v(\{2\})}{v(\{1\})+v(\{2\})} \cdot v(N)\right) .
$$

Thus in this case the worth of the grand coalition is distributed proportionally to the individual worths.

(iii) In general, fixed points for $h: X \rightarrow X$ need not exist, as one can easily check in the case of the following two-player game:

$$
N=\{1,2\}, \quad v(\{1\})=1, \quad v(\{2\})=-1, \quad v(N)=1 .
$$

However, we have the following first main result.

Theorem 1. There exists at least one balanced value for each monotone game. 
Let us indicate here only the main idea of the proof. (The full proof can be found in Section 4.) The first step is the result saying that if the game $(N, v)$ is monotone then the function $h$ redistributes each positive weight scheme $x \in X_{+}$to a positive weight scheme $h(x) \in X_{+}$(see Lemma 3(ii)). Then we define a multivalued mapping $F$ by assigning to each $x$ from the closure of $X^{+}$the convex hull of the set of all limit values of $\left.h\right|_{X_{+}}$at $x$. Applying Kakutani's fixed point theorem to $F$, we get a fixed point $x^{*}$ of $F$. Finally, we show that $x^{*}$ is also a fixed point of $h$. Note that we cannot apply directly Brouwer's fixed point theorem since the mapping $h$ is not continuous on $X$, as illustrated in Example 2 in the last section.

We refer to the solution that assigns to every game $(N, v) \in \mathcal{G}$ the set of balanced values $B(N, v)$ as the balanced solution. For monotone superadditive games, individual rationality seems a desirable property. It turns out that this property is satisfied by the balanced solution.

Theorem 2. If $(N, v)$ is a monotone superadditive game and $x$ is a balanced value of $(N, v)$, then $x_{i} \geq v(\{i\})$ for every $i \in N$.

The proof of this theorem can also be found in Section 4 .

Consider the game $(N, v)$ such that $v(S)=0$ for every $S \subsetneq N$ and $v(N)=1$. Then each $x \in X_{+}$is a balanced value. So we do not have uniqueness for balanced values even for the class of all monotone games. On the other hand, we do not know whether the balanced value is determined uniquely for any monotone or superadditive game $(N, w)$ with $w(\{i\})>0$ for every $i \in N$.

The next proposition captures properties of balanced values related to the symmetry property and the null player property.

Proposition 1. Let $(N, v)$ be a monotone game.

(i) If $i, j \in N, i \neq j$, are symmetric players in $(N, v)$, then there exists a balanced value $x^{*}$ of $(N, v)$ with $x_{i}^{*}=x_{j}^{*}$.

(ii) Let $i \in N$. If $v(\{i\})=0$, then there exists a balanced value $x^{*}$ of $(N, v)$ with $x_{i}^{*}=0$.

(iii) Let $i \in N$. If $v(\{i\})>0$, then each balanced value $x^{*}$ of $(N, v)$ satisfies $x_{i}^{*}>0$. 
(iv) If $k, l \in N$ are symmetric players with respect to $(N, v)$ and $v(\{k\})>0$ (and thus $v(\{l\})>0)$ then each balanced value $x^{*}$ of $(N, v)$ satisfies $x_{k}^{*}=x_{l}^{*}$.

It is well known that efficiency, the symmetry property, the null player property, and additivity uniquely determine the Shapley value. Let us remark that the balanced solution satisfies efficiency and the null player property on the class of all games, and the symmetry property on the class of all positive monotone games. (A game $(N, v)$ is positive if $v(S)>0$ for all nonempty $S \subseteq N$.)

Proposition 2. The balanced solution satisfies component efficiency, the component restriction property, and the null player property for the class $\mathcal{G}$.

Let us mention that balanced values need not be core allocations nor the other way around, as is demonstrated by the following example.

Example 1. Consider the game

$$
v(S)= \begin{cases}3 & \text { if } S=N, \\ 2 & \text { if }|S|=2, \\ 1 & \text { if } S=\{3\}, \\ 0 & \text { if } S \in\{\{1\},\{2\}\} .\end{cases}
$$

Note that Proposition 1(i) implies that there exists a balanced value with $x_{1}=x_{2}$. Further, Proposition 1(ii) implies that for players $i \in\{1,2\}$ there exists a balanced value with $x_{i}=0$. Since $v(\{1,2\})>0$ there cannot be a balanced value with $x_{1}=x_{2}=0$. Therefore we can conclude that there are at least three balanced values. It can be verified that $(0,3 / 2,3 / 2),(3 / 2,0,3 / 2)$, and $\frac{1}{10}(15-3 \sqrt{5}, 15-3 \sqrt{5}, 6 \sqrt{5})$ are the balanced values for $(N, v)$, while the core consists of the single point $(1,1,1)$.

However, for any monotone simple game $(N, v)$, every core allocation is a balanced value. Recall that a game $(N, v)$ is simple if $v(S) \in\{0,1\}$ for all $S \subseteq N$.

Proposition 3. If $(N, v)$ is a simple monotone game, then $\operatorname{Core}(N, v) \subseteq B(N, v)$.

Denote the class of all games with at least one balanced value by $\mathcal{G}_{B}$. We provide a characterization of the balanced solution among solutions defined on $\mathcal{G}_{B}$. We 
adapt the HM-reduced game property that characterizes the Shapley value in Hart and Mas-Colell $(1988,1989)$. The HM-reduced game property considers situations in which some players 'leave' the game. This property states that the payoffs of players that do not leave the game do not change if we consider an appropriately defined reduced game on the set of players remaining in the game. In this reduced game the worth of each coalition equals the worth of the union of this coalition and all players that leave the game minus the payoffs of the leaving players in the corresponding restricted game. The formal description is the following.

Let $(N, v) \in \mathcal{G}, f$ be a solution on the class $\mathcal{G}$, and $T \subseteq N$. The Hart and Mas-Colell reduced game $\left(T, \bar{v}_{T}^{f}\right)$ is given by

$$
\bar{v}_{T}^{f}(S)=v\left(S \cup T^{c}\right)-f_{T^{c}}\left(S \cup T^{c}, v_{S \cup T^{c}}\right) \text { for all } S \subseteq T,
$$

where $T^{c}=N \backslash T$ is the coalition of leaving players and $v_{S \cup T^{c}}$ is the restricted game on $S \cup T^{c}$ given by $v_{S \cup T^{c}}(H)=v(H)$ for all $H \subseteq S \cup T^{c}$.

A solution $f$ satisfies the HM-reduced game property if $f_{i}(N, v)=f_{i}\left(T, \bar{v}_{T}^{f}\right)$ for every $(N, v) \in \mathcal{G}, T \subseteq N$, and $i \in T$. Hart and Mas-Colell (1988) used the HMreduced game property to axiomatize the Shapley value.

To characterize the balanced solution we use a different reduced game which we refer to as the proportional reduced game. In this reduced game that results after some players have left the game, the dividend of a coalition $S$ of the remaining players is equal to the dividend of coalition $S$ in the original game plus a share in the original dividends of all coalitions containing $S$ and players who left the game. These shares are determined by the payoffs of the players in the original game. Here we have a formal definition.

Definition 4. Let $(N, v) \in \mathcal{G}, x \in X(N, v)$, and $T \subseteq N$ be nonempty. We define the proportional reduced game $\left(T, v_{T}^{x}\right)$ by determining its dividends

$$
\begin{aligned}
\Delta_{T, v_{T}^{x}}(S)=\Delta_{N, v}(S)+\sum_{\substack{K \subseteq T^{c}, K \neq \emptyset \\
x_{S} \cup K \neq 0}} \frac{x_{S}}{x_{S \cup K}} \Delta_{N, v}(S \cup K) \\
+\sum_{\substack{K \subseteq T^{c}, K \neq \emptyset \\
x_{S \cup K}=0}} \frac{|S|}{|S \cup K|} \Delta_{N, v}(S \cup K), \quad S \subseteq T .
\end{aligned}
$$


Definition 5. We say that a solution $F$ satisfies the proportional reduced game property on a class $\mathcal{C} \subseteq \mathcal{G}$ if, for every $(N, v) \in \mathcal{C}, x \in F(N, v)$, and nonempty $T \subseteq N$, we have $\left(T, v_{T}^{x}\right) \in \mathcal{C}$ and $\left.x\right|_{T} \in F\left(T, v_{T}^{x}\right)$.

Remark 3. The formula for $\Delta_{T, v_{T}^{x}}(S)$ can also be written as follows

$$
\Delta_{T, v_{T}^{x}}(S)=\sum_{\substack{K \subseteq T^{c} \\ x_{S \cup K} \neq 0}} \frac{x_{S}}{x_{S \cup K}} \Delta_{N, v}(S \cup K)+\sum_{\substack{K \subseteq T^{c} \\ x_{S \cup K}=0}} \frac{|S|}{|S \cup K|} \Delta_{N, v}(S \cup K) .
$$

Similarly to the HM-reduced game property, the proportional reduced game property can be seen as a consistency property. The just defined notions provide a 'partial axiomatization' of the balanced solution whose proof is contained in Section 4 .

Proposition 4. (i) The balanced solution satisfies the proportional reduced game property on $\mathcal{G}_{B}$.

(ii) If an efficient solution $F$ on $\mathcal{G}_{B}$ satisfies the proportional reduced game property on $\mathcal{G}_{B}$, then $F(N, v) \subseteq B(N, v)$ for every $(N, v) \in \mathcal{G}_{B}$.

Now we introduce the converse proportional reduced game property to obtain a characterization of the balanced solution.

Definition 6. A solution $F$ is said to satisfy the converse proportional reduced game property on a class $\mathcal{C}$ if, for every $(N, v) \in \mathcal{C}$ and every $x \in X(N, v)$ satisfying $v_{T}^{x} \in \mathcal{C}$ and $\left.x\right|_{T} \in F\left(T, v_{T}^{x}\right)$ for every nonempty $T \subsetneq N$, we have $x \in F(N, v)$.

A characterization of the balanced solution on $\mathcal{G}_{B}$ can be formulated as follows.

Theorem 3. A solution $F$ on $\mathcal{G}_{B}$ is efficient, satisfies the proportional reduced game property and the converse proportional reduced game property on $\mathcal{G}_{B}$ if and only if it is the balanced solution.

The proof again can be found in Section 4. Note that we characterize a set-valued solution, while Hart and Mas-Colell (1989) characterize a single-valued solution. Comparing our proportional reduced game with the HM-reduced game, we see that the latter takes account of payoffs of 'leaving' players in restricted games $\left(T, v_{T}\right)$, while what matters in our proportional reduced game are the payoffs of 'leaving' 
players in the original game $(N, v)$. Because the cardinality of player sets in restricted games is lower than the cardinality of the player set in the original game, the induction hypothesis in the proof of Hart and Mas-Colell (1989) uniquely determines the payoffs of players in the HM-reduced games. This cannot be done in the proof of Theorem 3 .

Hart and Mas-Colell (1989) characterize the Shapley value by their reduced game property and standardness for two player games meaning that in any two player game each player gets its own singleton worth plus half of the dividend of the two player 'grand' coalition. Note that the above given axioms that characterize the balanced solution on $\mathcal{G}_{B}$ imply proportional standardness for two player games meaning that in any two player game with positive singleton worths the worth of the two player 'grand' coalition is allocated proportionally to the singleton worths.

Hart and Mas-Colell (1989) also characterize weighted Shapley values by a 'weighted' standardness for two-player games property and the HM-reduced game property. However, their weighted Shapley values consider exogenously given weights which are the same for both the original and the reduced game. The balanced solution does not satisfy the HM-reduced game property because the endogenously determined balanced weights in the HM-reduced game (i.e., components $x_{i}$ of a balanced value $x$ in the HM-reduced game) can be different from the balanced weights in the original game.

\section{ProOFS}

4.1. Proof of Theorem 1. Some of the results presented in this subsection are standard (e.g., Lemma 4 and 5). We include them for the convenience of the reader.

Let $(N, v) \in \mathcal{G}$. The symbol $X_{0}(N, v)$ denotes the set of all $x \in X(N, v)$ with nonnegative coordinates. Usually we will write just $X_{0}$ instead of $X_{0}(N, v)$.

Let $S \subseteq N$ and $x \in \mathbb{R}^{N}$ be a nonnegative vector (i.e., all coordinates of $x$ are nonnegative) with $x_{S}>0$. We set

$$
k_{S}(x)=\sum_{\substack{T \subseteq N \\ T \supseteq S}} \frac{(-1)^{|T|-|S|}}{x_{T}}, \quad q_{S}(x)=\sum_{\substack{T \subseteq N \\ T \supseteq S}} \frac{(-1)^{|T|-|S|}}{x_{T}^{2}} .
$$


We establish properties of $h_{i}, k_{S}$, and $q_{S}$ needed in the sequel. In the proofs we will abbreviate notation $\Delta_{N, v}(T)$ to $\Delta(T)$ in case there is no confusion about the game we consider.

Lemma 1. Let $(N, v) \in \mathcal{G}$ and $i \in N$. Then

$$
h_{i}(x)=x_{i} \sum_{\substack{S \subseteq N \\ i \in S}} k_{S}(x)(v(S)-v(S \backslash\{i\}))
$$

for $x \in X_{0}$ with $x_{i}>0$.

Proof. It is known that the dividends can be expressed as

$$
\Delta(T)=\sum_{S \subseteq T}(-1)^{|T|-|S|} v(S), \quad T \subseteq N
$$

We can write

$$
\begin{aligned}
& h_{i}(x)=\sum_{\substack{T \subseteq N \\
i \in T}} \frac{x_{i}}{x_{T}} \Delta(T)=\sum_{\substack{T \subseteq N \\
i \in T}} \frac{x_{i}}{x_{T}}\left(\sum_{S \subseteq T}(-1)^{|T|-|S|} v(S)\right) \\
& =x_{i} \sum_{S \subseteq N} \sum_{\substack{T \subseteq N \\
T \supseteq \bar{S}, i \in T}}(-1)^{|T|-|S|} \frac{1}{x_{T}} v(S) \\
& =x_{i} \sum_{\substack{S \subseteq N \\
i \in S}} \sum_{\substack{T \subseteq N \\
T \supseteq \bar{S}, i \in T}}(-1)^{|T|-|S|} \frac{1}{x_{T}} v(S)+x_{i} \sum_{\substack{S \subseteq N \\
i \bar{\notin} S}} \sum_{\substack{T \subseteq N \\
T \supseteq \bar{S}, i \in T}}(-1)^{|T|-|S|} \frac{1}{x_{T}} v(S) \\
& =x_{i} \sum_{\substack{S \subseteq N \\
i \in S}} \sum_{\substack{T \subseteq N \\
T \supseteq S}}(-1)^{|T|-|S|} \frac{1}{x_{T}} v(S) \\
& +x_{i} \sum_{\substack{R \subseteq N \\
i \in R}} \sum_{T \subseteq N}(-1)^{|T|-(|R|-1)} \frac{1}{x_{T}} v(R \backslash\{i\}) \\
& =x_{i} \sum_{\substack{S \subseteq N \\
i \in S}} \sum_{\substack{T \subseteq N \\
T \supseteq S}}(-1)^{|T|-|S|} \frac{1}{x_{T}} v(S)-x_{i} \sum_{\substack{S \subseteq N \\
i \in S}} \sum_{\substack{T \subseteq N \\
T \supseteq S}}(-1)^{|T|-|S|} \frac{1}{x_{T}} v(S \backslash\{i\}) \\
& =x_{i} \sum_{\substack{S \subseteq N \\
i \in S}} k_{S}(x)(v(S)-v(S \backslash\{i\})) \text {. }
\end{aligned}
$$

Lemma 2. Let $N$ be a finite nonempty set, $S \subseteq N$, and $x \in \mathbb{R}^{N}$ be a nonnegative vector with $x_{S}>0$. Then we have $k_{S}(x)>0$ and $q_{S}(x)>0$.

Proof. We start with the following claim. 
Claim 1. Let $g$ be a positive $\mathcal{C}^{\infty}$ function on $(0,+\infty)$ such that $(-1)^{p} g^{(p)}$ is positive on $(0,+\infty)$ for every $p \in \mathbb{N}$. Then for every nonempty finite set $N, S \subseteq N$, and every nonnegative vector $x \in \mathbb{R}^{N}$ with $x_{S}>0$ we have

$$
\sum_{\substack{T \subseteq N \\ T \supseteq S}}(-1)^{|T|-|S|} g\left(x_{T}\right)>0
$$

Proof. Fix a nonempty finite set $N$ and denote $|N|=n$. If $|S|=|N|$, then the assertion obviously holds since $g$ is positive. Assume that the assertion is valid for this $N$ and every $g, S$, and $x$ satisfying the required properties and moreover $|S|>k$, where $k<|N|$.

We are going to prove the assertion for $S \subseteq N$ with $|S|=k$ and nonnegative $x \in \mathbb{R}^{N}$ with $x_{S}>0$. Take $i \in N \backslash S$. Define an auxiliary function $\psi$ by

$$
\psi(z)=\sum_{\substack{T \subseteq N \\ T \supseteq \bar{S} \cup\{i\}}}(-1)^{|T|-|S|} g\left(x_{T \backslash\{i\}}+z\right)+\sum_{\substack{T \subseteq N \\ T \supseteq \bar{S}, i \notin T}}(-1)^{|T|-|S|} g\left(x_{T}\right), \quad z \in[0, \infty) .
$$

We have

$$
\psi(0)=\sum_{\substack{L \subseteq N \\ L \supseteq \bar{S}, i \notin L}}-(-1)^{|L|-|S|} g\left(x_{L}\right)+\sum_{\substack{T \subseteq N \\ T \supseteq \bar{S}, i \notin T}}(-1)^{|T|-|S|} g\left(x_{T}\right)=0 .
$$

We compute the first derivative of $\psi$

$$
\psi^{\prime}(z)=\sum_{\substack{T \subseteq N \\ T \supseteq \bar{S} \cup\{i\}}}(-1)^{|T|-|S|} g^{\prime}\left(x_{T \backslash\{i\}}+z\right) .
$$

Set $P:=S \cup\{i\}$. Using the induction hypothesis, we obtain

$$
\psi^{\prime}(z)=\sum_{\substack{T \subseteq N \\ T \supseteq P}}(-1)^{|T|-|P|}\left(-g^{\prime}\right)\left(x_{T \backslash\{i\}}+z\right)>0 \quad \text { for } z \in[0,+\infty) .
$$

Here we have used the fact that the function $-g^{\prime}=(-1)^{1} g^{(1)}$ is positive and satisfies the required conditions on signs of its derivatives. Using (2) and (3), we obtain

$$
\psi\left(x_{i}\right)=\sum_{\substack{T \subseteq N \\ T \supseteq S}}(-1)^{|T|-|S|} g\left(x_{T}\right)>0
$$

and the claim is proved.

Applying Claim 1 to the functions $g(t)=1 / t$ and $g(t)=1 / t^{2}$ the proof of Lemma 2 is finished. 
Throughout this subsection and in subsections 4.2 and 4.3 , we will assume that the considered game $(N, v)$ is monotone and satisfies $v(N)>0$. Then we have that the corresponding set $X_{+}$is nonempty. If $v(N)=0$, then the assertions of Theorem 1, Theorem 2, and Proposition 1 are trivial or vacuous. Again, we denote $|N|$ by $n$.

Lemma 3. (i) The mapping $h$ is continuous on $X_{+}$.

(ii) We have $h(x) \in X_{0}$ for all $x \in X_{+}$.

Proof. The statement (i) is obvious. As for (ii), recall that $h(x) \in X$ for every $x \in X$ by Remark 1 . Using monotonicity of the game $(N, v)$, Lemma 1 and Lemma 2 , we see that $h_{i}(x) \geq 0$ for every $x \in X_{+}$and $i \in N$. Thus we have $h(x) \in X_{0}$ for $x \in X_{+}$.

Now we define the mapping $H: X_{0} \rightarrow 2^{X_{0}}$ by

$$
H(x)=\left\{\alpha \in \mathbb{R}^{n} \mid \exists\left(x^{j}\right) \subseteq X_{+}: x^{j} \rightarrow x, h\left(x^{j}\right) \rightarrow \alpha\right\}
$$

Lemma 4. (i) The set $\left\{(x, y) \in X_{0} \times X_{0} \mid y \in H(x)\right\}$ is closed.

(ii) The set $H(x)$ is a nonempty compact subset of $X_{0}$ for every $x \in X_{0}$.

(iii) We have $H(x)=\{h(x)\}$ for every $x \in X_{+}$.

Proof. (i) Take sequences $\left(x^{j}\right), x^{j} \in X_{0}$, and $\left(y^{j}\right)$ such that $x^{j} \rightarrow x \in X_{0}, y^{j} \in$ $H\left(x^{j}\right)$, and $y^{j} \rightarrow y$. For each $j \in \mathbb{N}$ there exists $z^{j} \in X_{+}$such that $\left\|z^{j}-x^{j}\right\|<1 / j$ and $\left\|h\left(z^{j}\right)-y^{j}\right\|<1 / j$. Then $z^{j} \rightarrow x$ and $h\left(z^{j}\right) \rightarrow y$. Consequently, $y \in H(x)$.

(ii) Fix $x \in X_{0}$. Since $X_{0}$ is compact, we have $H(x) \subseteq X_{0}$ by Lemma 3(ii). Using (i) and compactness of $X_{0}$, we get that $H(x)$ is compact. To prove that $H(x) \neq \emptyset$ take a sequence $\left(x^{j}\right), x^{j} \in X_{+}$, with $x^{j} \rightarrow x$. By Lemma 3(ii) the sequence $\left(h\left(x^{j}\right)\right)$ is contained in the compact set $X_{0}$. Therefore, there exists a convergent subsequence $\left(h\left(x^{j_{k}}\right)\right)_{k=1}^{\infty}$ with a limit $\alpha \in X_{0}$. Thus $\alpha \in H(x)$, showing that $H(x) \neq \emptyset$.

(iii) This follows from continuity of $h$ on $X_{+}$.

Now let us define a mapping $F$ from $X_{0}$ to the set of all convex subsets of $X_{0}$ such that $F(x)$ is the convex envelope of $H(x)$ for every $x \in X_{0}$.

Lemma 5. (i) The set $F(x)$ is a nonempty convex compact subset of $X_{0}$ for all $x \in X_{0}$. 
(ii) We have $F(x)=\{h(x)\}$ for every $x \in X_{+}$.

(iii) The set $\left\{(x, y) \in X_{0} \times X_{0} \mid y \in F(x)\right\}$ is closed.

Proof. (i) This assertion immediately follows from Lemma 4(ii), convexity of $X_{0}$, and the well-known fact that the convex envelope of any compact subset of $\mathbb{R}^{n}$ is compact.

(ii) This part clearly follows from Lemma 4(iii).

(iii) Take sequences $\left(x^{j}\right), x^{j} \in X_{0}$, and $\left(y^{j}\right)$ such that $x^{j} \rightarrow x \in X_{0}, y^{j} \in F\left(x^{j}\right)$, and $y^{j} \rightarrow y$. Now we use Carathéodory's theorem, which asserts that each element of the convex envelope of a set $M \subseteq \mathbb{R}^{n-1}$ can be written as a convex combination of $n$ elements of the set $M$. Since the simplex $X_{0}$ is $n-1$ dimensional, there are $\alpha_{1}^{j}, \ldots, \alpha_{n}^{j} \in[0,1]$ and $y^{j, 1}, \ldots, y^{j, n} \in H\left(x^{j}\right)$ such that

$$
\alpha_{1}^{j} y^{j, 1}+\cdots+\alpha_{n}^{j} y^{j, n}=y^{j} \quad \text { and } \quad \sum_{s=1}^{n} \alpha_{s}^{j}=1 .
$$

Going to subsequences, if necessary, we may assume that $\alpha_{s}^{j} \rightarrow \alpha_{s} \in[0,1]$, and $y^{j, s} \rightarrow y^{\infty, s}$. Then

$$
\alpha_{1} y^{\infty, 1}+\cdots+\alpha_{n} y^{\infty, n}=y \quad \text { and } \quad \sum_{s=1}^{n} \alpha_{s}=1 .
$$

Since the graph of $H$ is closed by Lemma 4(i), we have that $y^{\infty, s} \in H(x)$, and thus $y \in F(x)$.

Kakutani's theorem (see Kakutani, 1941, or, e.g., Franklin, 1980) states that any multivalued $F$ from a nonempty compact convex subset $D$ of $\mathbb{R}^{n}$ to itself such that the graph of $F$ is closed and $F(x)$ is convex, closed, and nonempty for all $x \in D$, has a fixed point, i.e., there exists an $x^{*} \in D$ such that $x^{*} \in F\left(x^{*}\right)$.

Since we have shown that $F$ and its domain $X_{0}$ satisfy the assumptions of Kakutani's theorem, we have the following lemma.

Lemma 6. There exists $x^{*} \in X_{0}$ such that $x^{*} \in F\left(x^{*}\right)$.

The next lemma shows the relationships between fixed points of $h$ and $F$.

Lemma 7. Let $x^{*} \in X_{0}$ be such that $x^{*} \in F\left(x^{*}\right)$. Then $h\left(x^{*}\right)=x^{*}$. Moreover, if we set $Q=\left\{i \in N \mid x_{i}^{*}=0\right\}$, then $v(Q)=0$. 
Proof. We distinguish two cases.

a) If $x^{*} \in X_{+}$then $x^{*} \in F\left(x^{*}\right)=\left\{h\left(x^{*}\right)\right\}$ (Lemma 5(ii)) and, consequently, $x^{*}=h\left(x^{*}\right)$.

b) Suppose now that $x^{*} \in X_{0} \backslash X_{+}$. From the definition of $F$ there are elements $z^{1}, \ldots, z^{p} \in H\left(x^{*}\right)$ and $\beta_{1}, \ldots, \beta_{p} \in(0,1]$ such that $\sum_{s=1}^{p} \beta_{s}=1$ and

$$
\beta_{1} z^{1}+\cdots+\beta_{p} z^{p}=x^{*}
$$

Denote $Q=\left\{i \in N \mid x_{i}^{*}=0\right\}$. Since $z_{i}^{j} \geq 0, j=1, \ldots, p$, the equation (4) guarantees that $z_{i}^{j}=0$ for every $i \in Q, j \in\{1, \ldots, p\}$. Let us simplify the notation by setting $z:=z^{1}$. Since $z \in H\left(x^{*}\right)$ there exists a sequence $\left(x^{j}\right), x^{j} \in X_{+}$, such that $x^{j} \rightarrow x^{*}$ and $h\left(x^{j}\right) \rightarrow z$.

For $i \in Q$ we have $\lim _{j \rightarrow \infty} x_{i}^{j}=0$ and $\lim _{j \rightarrow \infty} h_{i}\left(x^{j}\right)=0$. Further, we get

$$
\begin{aligned}
\sum_{i \in Q} h_{i}\left(x^{j}\right) & =\sum_{i \in Q}\left(\sum_{S \subseteq N, i \in S} \frac{x_{i}^{j}}{x_{S}^{j}} \Delta(S)\right) \\
& =\sum_{i \in Q}\left(\left(\sum_{\substack{S \subseteq N, i \in S, S \backslash Q \neq \emptyset}} \frac{x_{i}^{j}}{x_{S}^{j}} \Delta(S)\right)+\left(\sum_{S \subseteq Q, i \in S} \frac{x_{i}^{j}}{x_{S}^{j}} \Delta(S)\right)\right) \\
& =\sum_{i \in Q}\left(\sum_{\substack{S \subseteq N \\
i \in S, S \backslash Q \neq \emptyset}} \frac{x_{i}^{j}}{x_{S}^{j}} \Delta(S)\right)+\sum_{i \in Q}\left(\sum_{S \subseteq Q, i \in S} \frac{x_{i}^{j}}{x_{S}^{j}} \Delta(S)\right) \\
& =\sum_{i \in Q}\left(\sum_{\substack{S \subseteq N \\
i \in S, S \backslash Q \neq \emptyset}} \frac{x_{i}^{j}}{x_{S}^{j}} \Delta(S)\right)+\left(\sum_{S \subseteq Q} \Delta(S)\right) \\
& =\sum_{i \in Q}\left(\sum_{\substack{S \subseteq N \\
i \in S, S \backslash Q \neq \emptyset}} \frac{x_{i}^{j}}{x_{S}^{j}} \Delta(S)\right)+v(Q) .
\end{aligned}
$$

The limit of the left side is

$$
\lim _{j \rightarrow \infty} \sum_{i \in Q} h_{i}\left(x^{j}\right)=0
$$


and, since $S \backslash Q \neq \emptyset$ implies $x_{S}^{*}>0$, we have also

$$
\lim _{j \rightarrow \infty} \sum_{i \in Q}\left(\sum_{\substack{S \subseteq N \\ i \in S, S \backslash Q \neq \emptyset}} \frac{x_{i}^{j}}{x_{S}^{j}} \Delta(S)\right)=0 .
$$

Therefore, we have $v(Q)=0$. This proves the second part of our statement.

By monotonicity of $(N, v)$ we get $v(S)=0$ for every $S \subseteq Q$. Consequently, $\Delta(S)=0$ for all $S \subseteq Q$. Then

$$
\begin{aligned}
h_{i}(x) & =\sum_{\substack{S \subseteq N \\
i \in S, x_{S} \neq 0}} \frac{x_{i}}{x_{S}} \Delta(S)+\sum_{\substack{S \subseteq N \\
i \in S, x_{S}=0}} \frac{1}{|S|} \Delta(S) \\
& =\sum_{\substack{S \subseteq N, i \in S \\
S \backslash Q \neq \emptyset, x_{S} \neq 0}} \frac{x_{i}}{x_{S}} \Delta(S)+\sum_{\substack{S \subseteq N, i \in S \\
S \backslash Q \neq \emptyset, x_{S}=0}} \frac{1}{|S|} \Delta(S), \quad x \in X_{0} .
\end{aligned}
$$

Since $x_{i}^{*}>0$ for all $i \in N \backslash Q$, there exists a neighborhood $V$ of $x^{*}$ such that, for every $x \in X_{0} \cap V$ and $S \subseteq N$ with $S \backslash Q \neq \emptyset$, we have $x_{S}>0$ and

$$
h_{i}(x)=\sum_{\substack{S \subseteq N, i \in S \\ S \backslash Q \neq \emptyset, x_{S} \neq 0}} \frac{x_{i}}{x_{S}} \Delta(S) .
$$

From this we conclude that $h$ is continuous at $x^{*}$.

From the continuity of $h$ at fixed point $x^{*}$ of $F$ it follows that $x^{*} \in F\left(x^{*}\right)=$ $\left\{h\left(x^{*}\right)\right\}$, and thus $x^{*}=h\left(x^{*}\right)$.

Now we immediately see that the assertion of Theorem 1 holds.

Note that we could not apply directly Brouwer's fixed point theorem since the mapping $h(N, v)$ is not continuous on $X(N, v)$, as illustrated in the following example.

Example 2. Consider the monotone game $(N, v)$ given by $N=\{1,2,3\}$ with the dividends

$$
\Delta_{N, v}(S)= \begin{cases}1 & \text { if } S \in\{\{3\},\{1,2\}\} \\ 0 & \text { otherwise }\end{cases}
$$

and $x^{\varepsilon}=(\varepsilon, 2 \varepsilon, 2-3 \varepsilon)$. Clearly, $h\left(x^{\varepsilon}\right)=(1 / 3,2 / 3,1)$ whenever $\varepsilon \in(0,2 / 3)$ and $h\left(x^{\varepsilon}\right)=(1 / 2,1 / 2,1)$ for $\varepsilon=0$. 


\subsection{Proof of Theorem 2.}

Lemma 8. For every $x \in X_{0}$ we have $h(x) \in F(x)$.

Proof. Denote $Q=\left\{i \in N \mid x_{i}=0\right\}$ and for $\varepsilon>0$ we set

$$
y_{i}^{\varepsilon}= \begin{cases}\varepsilon & \text { for } i \in Q ; \\ x_{i}-\frac{|Q|}{|N|-|Q|} \varepsilon & \text { for } i \in N \backslash Q .\end{cases}
$$

If $\varepsilon>0$ is sufficiently small, then $y^{\varepsilon} \in X_{+}$and we have

$$
h_{i}\left(y^{\varepsilon}\right)=\sum_{\substack{S \subseteq N \\ i \in S}} \frac{y_{i}^{\varepsilon}}{y_{S}^{\varepsilon}} \Delta(S)=\sum_{\substack{S \subseteq N \\ i \in S, x_{S} \neq 0}} \frac{y_{i}^{\varepsilon}}{y_{S}^{\varepsilon}} \Delta(S)+\sum_{\substack{S \subseteq N \\ i \in S, x_{S}=0}} \frac{\varepsilon}{|S| \varepsilon} \Delta(S), \quad i \in Q
$$

Now it is easy to see that $h\left(y^{\varepsilon}\right) \rightarrow h(x)$ for $\varepsilon \rightarrow 0+$. This shows $h(x) \in H(x) \subseteq F(x)$ and we are done.

Now we prove Theorem 2. By Theorem 1 , the set $B(N, v)$ is nonempty. Using Lemma 1 and superadditivity of $(N, v)$, we get for $x \in X_{0}$ with $x_{i}>0$ the following estimates.

$$
\begin{aligned}
h_{i}(x) & =x_{i} \sum_{\substack{S \subseteq N \\
i \in S}} k_{S}(x)(v(S)-v(S \backslash\{i\})) \\
& \geq x_{i} \sum_{\substack{S \subseteq N \\
i \in S}}\left(k_{S}(x) \cdot v(\{i\})\right)=x_{i} \cdot v(\{i\}) \cdot \sum_{\substack{S \subseteq N \\
i \in S}} k_{S}(x) .
\end{aligned}
$$

The term $\sum_{\substack{S \subseteq N \\ i \in S}} k_{S}(x)$ can be rewritten as follows

$$
\begin{aligned}
\sum_{\substack{S \subseteq N \\
i \in S}} k_{S}(x) & =\sum_{\substack{S \subseteq N \\
i \in S}} \sum_{\substack{T \subseteq N \\
T \supseteq S}} \frac{(-1)^{|T|-|S|}}{x_{T}} \\
& =\sum_{\substack{T \subseteq N \\
i \in T}} \sum_{\substack{S \subseteq T \\
i \in S}} \frac{(-1)^{|T|-|S|}}{x_{T}} \\
& =\sum_{\substack{T \subseteq N \\
i \in T}}\left(\frac{(-1)^{|T|}}{x_{T}} \cdot \sum_{S \subseteq T, i \in S}(-1)^{-|S|}\right) .
\end{aligned}
$$


Further, we compute

$$
\begin{aligned}
\sum_{S \subseteq T, i \in S}(-1)^{-|S|} & =\sum_{R \subseteq T \backslash\{i\}}(-1)^{-|R|-1}=-\sum_{n=0}^{|T|-1} \sum_{\substack{R \subseteq T \backslash\{i\} \\
|R|=n}}(-1)^{-n} \\
& =-\left(\left(\begin{array}{c}
|T|-1 \\
0
\end{array}\right)-\left(\begin{array}{c}
|T|-1 \\
1
\end{array}\right)+\cdots+(-1)^{|T|-1}\left(\begin{array}{c}
|T|-1 \\
|T|-1
\end{array}\right)\right) \\
& =\left\{\begin{array}{cc}
-1 & \text { for }|T|=1, \\
-(1-1)^{|T|-1}=0 & \text { for }|T|>1 .
\end{array}\right.
\end{aligned}
$$

Thus we get

$$
\sum_{\substack{S \subseteq N \\ i \in S}} k_{S}(x)=\sum_{\substack{T \subseteq N \\ i \in T,|T|=1}} \frac{(-1)^{|T|+1}}{x_{T}}=\frac{1}{x_{i}} .
$$

The inequality (5) and the identity (6) yield $h_{i}(x) \geq v(\{i\})$ for $x \in X_{0}$ with $x_{i}>0$.

Now let $x^{*}$ be a balanced value of the game $(N, v)$. Denote $Q=\left\{i \in N \mid x_{i}^{*}=0\right\}$. If $i \in N \backslash Q$, then we have $x_{i}^{*}>0$ and so $x_{i}^{*}=h_{i}\left(x^{*}\right) \geq v(\{i\})$ as shown above.

By Lemmas 7 and 8 we have $v(Q)=0$. By monotonicity this leads to $v(\{i\})=0$ for all $i \in Q$, and we have $x_{i}^{*}=v(\{i\})$ for every $i \in Q$, showing individual rationality of the balanced solution for monotone superadditive games.

4.3. Proof of Proposition 1. We again use the same notation as in the previous subsections.

(i) Set $Z=\left\{x \in X_{0} \mid x_{i}=x_{j}\right\}$. The set $Z$ is nonempty, compact, and convex. Let $F$ be the mapping defined in subsection 4.1 after Lemma 4 . We define a multivalued mapping $G$ by $G(x)=F(x) \cap Z$. Using the symmetry of the players $i$ and $j$, we have that $h_{i}(x)=h_{j}(x)$ whenever $x \in Z$. This and Lemma 8 implies that $h(x) \in G(x)$ for every $x \in Z$. Consequently, $G(x) \neq \emptyset$ for every $x \in Z$. Further, it is clear that $G(x)$ is a compact convex set and the set $\{(x, y) \in Z \times Z \mid y \in G(x)\}$ is closed (see Lemma 5). Applying Kakutani's theorem, we get a fixed point $x^{*} \in Z$ of the mapping $G$. Then clearly $x^{*}$ is a fixed point of $F$ and $x_{i}^{*}=x_{j}^{*}$. By Lemma 7 we get that $x^{*}$ is a fixed point of $h$.

(ii) The idea of this proof is the same as in the previous part. Set $R=\left\{x \in X_{0} \mid\right.$ $\left.x_{i}=0\right\}$. The set $R$ is nonempty, compact, and convex. Let $F$ again be the mapping 
defined in subsection 4.1. We define a multivalued mapping $G$ by $G(x)=F(x) \cap R$, $x \in R$. It is clear that $G(x)$ is a compact convex set for every $x \in R$ and the set $\{(x, y) \in R \times R \mid y \in G(x)\}$ is closed.

We also show that $G(x)$ is nonempty whenever $x \in R$. To this end, fix $x \in R$. Since $v(N)>0$, one can find $j \in N$ with $x_{j}>0$. We define $y^{\varepsilon} \in X$ by

$$
y_{k}^{\varepsilon}= \begin{cases}\varepsilon^{2} & \text { for } k=i ; \\ x_{k}+\varepsilon & \text { for } k \in N \backslash\{i, j\} ; \\ x_{j}-\varepsilon^{2}-(|N|-2) \varepsilon & \text { for } k=j .\end{cases}
$$

For every $\varepsilon \geq 0$ we have $y^{\varepsilon} \in X$ and for sufficiently small $\varepsilon>0$ we have $y^{\varepsilon} \in$ $X_{+}$. Then we have $\lim _{\varepsilon \rightarrow 0+} y^{\varepsilon}=x$, and a straightforward computation results in $\lim _{\varepsilon \rightarrow 0+} y_{i}^{\varepsilon} / y_{S}^{\varepsilon}=0$ for every nonempty $S \subseteq N$ with $S \neq\{i\}$. Using this and $\Delta(\{i\})=0$, we infer $\lim _{\varepsilon \rightarrow 0+} h_{i}\left(y^{\varepsilon}\right)=0$. This implies that there exists a sequence $\left(y^{j}\right)$ of elements of $X_{+}$going to $x$ such that $\left(h\left(y^{j}\right)\right)_{j}$ converges to some $\alpha \in X_{0}$ with $\alpha_{i}=0$. Thus $\alpha \in F(x) \cap R$ and $G(x) \neq \emptyset$.

Applying Kakutani's theorem, we obtain a fixed point $x^{*} \in R$ of the mapping $G$. Thus $x_{i}^{*}=0$ and $x^{*} \in F\left(x^{*}\right)$. By Lemma 7 we get that $x^{*}$ is a fixed point of $h$.

(iii) Suppose that $v(\{i\})>0$ and $x^{*}$ is a balanced value of $(N, v)$. Applying Lemma 8, we get $x^{*} \in F\left(x^{*}\right)$. Now from Lemma 7 it follows that $x_{i}^{*}>0$ since otherwise $v(\{i\})=0$.

(iv) Suppose on the contrary that there exists a balanced value $x^{*}$ of $(N, v)$ with $x_{k}^{*} \neq x_{l}^{*}$. Without any loss of generality we may assume that $k=1, l=2$, and $x_{1}^{*}<x_{2}^{*}$. Define $y^{*} \in X_{0}$ by $y_{1}^{*}=x_{2}^{*}, y_{2}^{*}=x_{1}^{*}$, and $y_{i}^{*}=x_{i}^{*}, i \in N \backslash\{1,2\}$. Because of the symmetry of players 1 and 2 we have $h_{1}\left(y^{*}\right)=h_{2}\left(x^{*}\right)=y_{1}^{*}$.

For $t \in \mathbb{R}$ set $z(t)=(1-t) x^{*}+t y^{*}$ and define an auxiliary function $g$ on $[0,1]$ by

$$
g(t)=\sum_{\substack{S \subseteq N \\ 1 \in S}} k_{S}(z(t))(v(S)-v(S \backslash\{1\})) .
$$

Since $v(\{1\})>0$ and $v(\{2\})>0$, we have $x_{1}^{*}>0$ and $x_{2}^{*}>0$ by Proposition 1(iii). Then we have $g(0)=h_{1}\left(x^{*}\right) / x_{1}^{*}=1$ and $g(1)=h_{1}\left(y^{*}\right) / y_{1}^{*}=h_{2}\left(x^{*}\right) / x_{2}^{*}=1$. Further, 
we compute

$$
g^{\prime}(t)=\left(x_{2}^{*}-x_{1}^{*}\right) \sum_{\substack{S \subseteq N \\ 1 \in S}}\left(\frac{\partial k_{S}}{\partial x_{1}}(z(t))-\frac{\partial k_{S}}{\partial x_{2}}(z(t))\right)(v(S)-v(S \backslash\{1\})) .
$$

For $S \subseteq N$ with $1 \in S$ and $x \in X_{0}$ with $x_{S}>0$ we have

$$
\begin{aligned}
\frac{\partial k_{S}}{\partial x_{1}}(x)-\frac{\partial k_{S}}{\partial x_{2}}(x) & =\sum_{\substack{T \subseteq N \\
T \supseteq S}}(-1)^{|T|-|S|}\left(-x_{T}^{-2}\right)-\sum_{\substack{T \subseteq N \\
T \supseteq \bar{S}, 2 \in T}}(-1)^{|T|-|S|}\left(-x_{T}^{-2}\right) \\
& =\sum_{\substack{T \subseteq N \\
T \supseteq \bar{S}, 2 \notin T}}(-1)^{|T|-|S|}\left(-x_{T}^{-2}\right)=-\sum_{\substack{T \subseteq N \backslash\{2\} \\
T \supseteq S}}(-1)^{|T|-|S|} x_{T}^{-2}
\end{aligned}
$$

If $2 \in S$ then the last term is 0 , otherwise it is negative by Lemma 2 . Since $x_{2}^{*}-x_{1}^{*}>0$, we thus get $g^{\prime} \leq 0$ on $(0,1)$. But we know also that

$$
\left(x_{2}^{*}-x_{1}^{*}\right)\left(\frac{\partial k_{\{1\}}}{\partial x_{1}}(x)-\frac{\partial k_{\{1\}}}{\partial x_{2}}(x)\right)(v(\{1\})-v(\emptyset))<0, \quad x \in X_{0}, x_{1}>0,
$$

and this yields $g^{\prime}<0$ on $(0,1)$. This is in contradiction with $g(0)=g(1)=1$.

4.4. Proof of Proposition 2. We start with the following claim.

Claim 2. Let $(N, v) \in \mathcal{G}, C$ be a component of $(N, v)$, and $i \in C$. Then

$$
h_{i}(N, v)(y)=h_{i}\left(C, v_{C}\right)\left(\left.y\right|_{C}\right) \quad \text { for every } y \in X(N, v) \text {, }
$$

where $\left(C, v_{C}\right)$ is the restriction of $(N, v)$ to $C$, i.e., $v_{C}(S)=v(S \cap C)$ for every $S \subseteq C$.

Proof. Firts of all, we prove that if $S \subseteq N$ intersects both $C$ and $N \backslash C$, then $\Delta(S)=0$. If $S$ contains just two elements, then the observation follows directly from the definition of component. Now take a set $S$ with the required property and assume that the observation holds for all sets having less elements than $S$. Using this assumption and the definition of component we have

$$
\begin{aligned}
\Delta(S) & =v(S)-\sum_{T \subsetneq S} \Delta(T)=v(S)-\sum_{T \subseteq S \cap C} \Delta(T)-\sum_{T \subseteq S \backslash C} \Delta(T) \\
& =v(S)-v(S \cap C)-v(S \backslash C)=0 .
\end{aligned}
$$

Then the assertion of Claim 2 follows immediately from the observation that $\Delta_{(N, v)}(S)=\Delta_{\left(C, v_{C}\right)}(S)$ if $S \subseteq C$. 
We will continue with the proof of Proposition 2. To prove component efficiency consider $(N, v) \in \mathcal{G}$ with a component $C$ and $x \in B(N, v)$. Using Claim 2 we get

$$
x_{C}=\sum_{i \in C} h_{i}(N, v)(x)=\sum_{i \in C} h_{i}\left(C, v_{C}\right)\left(\left.x\right|_{C}\right)=v(C) .
$$

The last equality follows from Remark 1.

Now consider $x, x^{\prime} \in B(N, v)$ and a vector $x^{\prime \prime}$ defined by

$$
x_{i}^{\prime \prime}= \begin{cases}x_{i} & \text { for } i \in C, \\ x_{i}^{\prime} & \text { for } i \in N \backslash C .\end{cases}
$$

We show that $x^{\prime \prime} \in B(N, v)$. According to component efficiency we have

$$
x_{N}^{\prime \prime}=x_{C}^{\prime \prime}+x_{N \backslash C}^{\prime \prime}=x_{C}+x_{N \backslash C}^{\prime}=v(C)+v(N \backslash C)=v(N) .
$$

Now it is clear that $x^{\prime \prime} \in X_{0}(N, v)$. Using Claim 2, for $i \in C$, we have

$$
x_{i}^{\prime \prime}=x_{i}=h_{i}(N, v)(x)=h_{i}\left(C, v_{C}\right)\left(\left.x\right|_{C}\right)=h_{i}\left(C, v_{C}\right)\left(\left.x^{\prime \prime}\right|_{C}\right)=h_{i}(N, v)\left(x^{\prime \prime}\right) .
$$

The equality $x_{i}^{\prime \prime}=h_{i}\left(x^{\prime \prime}\right)$ for $i \in N \backslash C$ immediately follows since $N \backslash C$ is a component of $(N, v)$ as well.

The null player property of the balanced solution follows from component efficiency since $C:=\{i\}$, where $i$ is a null player in $(N, v)$, is a component in $(N, v)$.

4.5. Proof of Proposition 3. Let $(N, v)$ be a simple monotone game. Suppose that $x \in \operatorname{Core}(N, v)$. Then there exists a coalition $T \subseteq N$ such that $v(S)=1$ if and only if $T \subseteq S \subseteq N$.

If $\Delta(S) \neq 0$, then $x_{S}=1$. Indeed, $\Delta(S) \neq 0$ implies $v(S) \neq 0$. This yields $T \subseteq S$ and $1 \geq x_{S} \geq x_{T} \geq v(T)=1$, showing $x_{S}=1$.

Applying this observation, we get

$$
h_{i}(x)=\sum_{\substack{S \subseteq N, i \in S \\ x_{S}=1}} \frac{x_{i}}{x_{S}} \Delta(S)=x_{i} \sum_{\substack{S \subseteq N, i \in S \\ x_{S}=1}} \Delta(S)=x_{i} \sum_{S \subseteq N, i \in S} \Delta(S), \quad i \in N
$$

The last equality holds since we added just the zero dividends. Since the last sum equals $v(N)=1$, we have $h_{i}(x)=x_{i}$ and we are done. 
4.6. Proof of Proposition 4. The following lemma will be useful in the sequel.

Lemma 9. Let $(N, v) \in \mathcal{G}_{B}, x \in B(N, v)$, and $T \subseteq N$ be nonempty. Then we have $h_{i}\left(T, v_{T}^{x}\right)\left(\left.x\right|_{T}\right)=h_{i}(N, v)(x)$ for every $i \in T$.

Proof. By the definition of $\Delta_{T, v_{T}^{x}}$ we have

$$
\begin{aligned}
\sum_{\substack{S \subseteq T, i \in S \\
x_{S} \neq 0}} \frac{x_{i}}{x_{S}} \Delta_{T, v_{T}^{x}}(S) & =\sum_{\substack{S \subseteq T, i \in S \\
x_{S} \neq 0}} \sum_{\substack{K \subseteq T^{c} \\
x_{S \cup K} \neq 0}} \frac{x_{i}}{x_{S}} \cdot \frac{x_{S}}{x_{S \cup K}} \Delta_{N, v}(S \cup K) \\
& +\sum_{\substack{S \subseteq T, i \in S \\
x_{S} \neq 0}} \sum_{\substack{K \subseteq T^{c} \\
x_{S} \cup K=0}} \frac{x_{i}}{x_{S}} \cdot \frac{|S|}{|S \cup K|} \Delta_{N, v}(S \cup K) .
\end{aligned}
$$

For $x \in X_{0}(N, v)$ and $S \subseteq N, x_{S}=0$ implies that $x_{i}=0$ for all $i \in S$. It follows that $x_{S \cup K}=0$ implies $x_{S}=0$; thus the last term in the expression above disappears so we can write

$$
\begin{aligned}
\sum_{\substack{S \subseteq T, i \in S \\
x_{S} \neq 0}} \frac{x_{i}}{x_{S}} \Delta_{T, v_{T}^{x}}(S) & =\sum_{\substack{S \subseteq T, i \in S \\
x_{S} \neq 0}} \sum_{\substack{K \subseteq T^{c} \\
x_{S \cup K} \neq 0}} \frac{x_{i}}{x_{S \cup K}} \Delta_{N, v}(S \cup K) \\
& =\sum_{\substack{S \subseteq T, i \in S \\
x_{S} \neq 0}} \sum_{K \subseteq T^{c}} \frac{x_{i}}{x_{S \cup K}} \Delta_{N, v}(S \cup K) .
\end{aligned}
$$

Similarly, we can write

$$
\begin{aligned}
\sum_{\substack{S \subseteq T, i \in S \\
x_{S}=0}} \frac{1}{|S|} \Delta_{T, v_{T}^{x}}(S) & =\sum_{\substack{S \subseteq T, i \in S \\
x_{S}=0}} \sum_{\substack{K \subseteq T^{c} \\
x_{S \cup K} \neq 0}} \frac{1}{|S|} \cdot \frac{x_{S}}{x_{S \cup K}} \Delta_{N, v}(S \cup K) \\
& +\sum_{\substack{S \subseteq T, i \in S \\
x_{S}=0}} \sum_{\substack{K \subseteq T^{c} \\
x_{S \cup K}=0}} \frac{1}{|S|} \cdot \frac{|S|}{|S \cup K|} \Delta_{N, v}(S \cup K) \\
& =\sum_{\substack{S \subseteq T, i \in S \\
x_{S}=0}} \sum_{\substack{K \subseteq T^{c} \\
x_{K} \neq 0}} \frac{1}{|S|} \cdot \frac{x_{S}}{x_{S \cup K}} \Delta_{N, v}(S \cup K) \\
& +\sum_{\substack{S \subseteq T, i \in S \\
x_{S}=0}} \sum_{\substack{K \subseteq T^{c} \\
x_{K}=0}} \frac{1}{|S \cup K|} \Delta_{N, v}(S \cup K) .
\end{aligned}
$$

In the expression above we have $x_{S}=0$ and $i \in S$. Thus $x_{i}=0$, and we can replace the zero term $\frac{1}{|S|} \cdot \frac{x_{S}}{x_{S \cup K}}$ by another zero term $\frac{x_{i}}{x_{S \cup K}}$. Thus we have 


$$
\begin{aligned}
\sum_{\substack{S \subseteq T, i \in S \\
x_{S}=0}} \frac{1}{|S|} \Delta_{T, v_{T}^{x}}(S) & =\sum_{\substack{S \subseteq T, i \in S \\
x_{S}=0}} \sum_{\substack{K \subseteq T^{c} \\
x_{K} \neq 0}} \frac{x_{i}}{x_{S \cup K}} \Delta_{N, v}(S \cup K) \\
& +\sum_{\substack{S \subseteq T, i \in S \\
x_{S}=0}} \sum_{\substack{K \subseteq T^{c} \\
x_{K}=0}} \frac{1}{|S \cup K|} \Delta_{N, v}(S \cup K) .
\end{aligned}
$$

Summing up (8) and (9) provides the desired equality.

Now we can start with the proof of Proposition 3.

(i) The desired assertion follows easily from Lemma 9.

(ii) We will proceed by induction on the cardinality of $N$. If $|N|=1$, then $F(N, v)=B(N, v)$ by efficiency of $F$ and the balanced solution. Let $(N, v) \in$ $\mathcal{G}_{B},|N|>1$, and suppose that $F\left(N^{\prime}, v^{\prime}\right) \subseteq B\left(N^{\prime}, v^{\prime}\right)$ for any $\left(N^{\prime}, v^{\prime}\right) \in \mathcal{G}_{B}$ with $\left|N^{\prime}\right|<|N|$. Let $i \in N$. Suppose that $x \in F(N, v)$. The proportional reduced game property of $F$ on $\mathcal{G}_{B}$ implies that $v_{\{i\}}^{x} \in \mathcal{G}_{B}$ and $\left.x\right|_{\{i\}} \in F\left(\{i\}, v_{\{i\}}^{x}\right)$. By the induction hypothesis we have $\left.x\right|_{\{i\}} \in B\left(\{i\}, v_{\{i\}}^{x}\right)$. Using Lemma 9, we get $x_{i}=h_{i}\left(\{i\}, v_{\{i\}}^{x}\right)\left(\left.x\right|_{\{i\}}\right)=h_{i}(N, v)(x)$. Since $i \in N$ has been chosen arbitrarily, we have $x \in B(N, v)$.

4.7. Proof of Theorem 3. First we show that the balanced solution satisfies the converse proportional reduced game property. Take $(N, v) \in \mathcal{G}_{B}$ and let $x \in X(N, v)$ be such that $\left.x\right|_{T} \in B\left(T, v_{T}^{x}\right)$ for all nonempty $T \subsetneq N$. Set $T=\{i\}, i \in N$. By Lemma 9 we have

$$
x_{i}=h_{i}\left(\{i\}, v_{\{i\}}^{x}\right)\left(\left.x\right|_{\{i\}}\right)=h_{i}(N, v)(x) .
$$

It remains to show that, if an efficient solution $F$ satisfies the proportional reduced game property and the converse proportional reduced game property, then $F$ is the balanced solution. Such an $F$ coincides with the balanced solution on the class of one-person games from $\mathcal{G}_{B}$ by efficiency. We will proceed by induction on the cardinality of $N$. Assume that $F\left(N^{\prime}, v^{\prime}\right)=B\left(N^{\prime}, v^{\prime}\right)$ for every $\left(N^{\prime}, v^{\prime}\right) \in \mathcal{G}_{B}$ with $\left|N^{\prime}\right|<|N|$. By Proposition 4(ii) we have that $F(N, v) \subseteq B(N, v)$. So we are left to show that $B(N, v) \subseteq F(N, v)$.

Suppose that $x \in B(N, v)$. Since $B$ satisfies the proportional reduced game property, it follows that $\left.x\right|_{T} \in B\left(T, v_{T}^{x}\right)$ for all nonempty $T \subsetneq N$. By the induction 
hypothesis $B\left(T, v_{T}^{x}\right)=F\left(T, v_{T}^{x}\right)$, and thus $\left.x\right|_{T} \in F\left(T, v_{T}^{x}\right)$ for all nonempty $T \subsetneq N$. The converse proportional reduced game property of $F$ implies that $x \in F(N, v)$ and the proof is finished.

\section{REFERENCES}

Aumann, R. J., And J. H. Drèze (1980): "Cooperative Games with Coalition Structures," International Journal of Game Theory, 3, 217-237.

Chang, C., And C.-Y. Kan (1994): "A Study on Decomposable Convex Games," Games and Economic Behavior, 7, 35-38.

Feldman, B. (2005): "The Proportional Value of a Cooperative Game," Manuscript: Scuder Kemper Investment.

Franklin, J. (1980): Methods of mathematical economics. Linear and nonlinear programming, fixed-point theorems. Undergraduate Texts in Mathematics. Springer-Verlag, New York-Berlin.

Harsanyi, J. C. (1959): "A Bargaining Model for Cooperative n-Person Games," in Contributions to the Theory of Games IV, ed. by A. W. Tucker, and R. D. Luce, pp. 325-355. Princeton University Press, Princeton.

Hart, S., And A. Mas-Colell (1988): "The Potential of the Shapley Value," in The Shapley Value. Essays in Honor of L.S. Shapley, ed. by A. E. Roth, pp. 127-137. Cambridge University Press, Cambridge.

(1989): "Potential, Value and Consistency," Econometrica, 57, 589-614.

Homans, C. G. (1961): Social behavior: its elementary forms. Routledge \& Paul, London.

Kakutani, S. (1941): "A generalization of Brouwer's fixed point theorem," Duke Math. J., 8, $457-459$.

KalaI, E. (1977): "Proportional Solutions to Bargaining Situations: Interpersonal Utility Comparisons," Econometrica, 45, 1623-1630.

Ortmann, K. M. (2000): "The Proportional Value for Positive Cooperative Games," Mathematical Methods of Operations Research, 51, 235-248.

Roth, A. E. (1979): "Proportional Solutions to the Bargaining Problem," Econometrica, 47, $775-778$.

Selten, R. (1978): "The equity principle in economic behavior," in Decision Theory and Social Ethics: Issues in Social Choice, ed. by H. Gottinger, and W. Leinfellner, pp. 289-301. D. Reidel Publishing Company, Dordrecht, Holland.

Shapley, L. S. (1953a): Additive and Non-Additive Set Functions. Dissertation, Princeton University, Princeton. 
(1953b): "A Value for n-Person Games," in Contributions to the Theory of Games II, ed. by H. W. Kuhn, and A. W. Tucker, pp. 307-317. Princeton University Press, Princeton.

Vorob'ev, N. N., And A. V. Liapounov (1998): “The Proper Shapley Value,” in Game Theory and Applications Vol IV, ed. by L. Petrosyan, and M. Mazalov, pp. 155-159. Nova Science, New York. 